\title{
Electronic speckle pattern surface contouring using optical fibres and wavelength modulation of laser diodes
}

\author{
Ralph P Tatam \\ School of Mechanical Engineering, Cranfield Institute of \\ Technology, Bedford MK43 OAL, U.K. \\ Jeremy C Davies, Clive H Buckberry \\ Test Techniques Development, Rover Group plc, Lighthorne, \\ Warwickshire, CV35 OBL, U.K. \\ Julian D C Jones \\ Physics Department, Heriot-Watt University, Riccarton, \\ Edinburgh, EH14 4AS, U.K.
}

\begin{abstract}
The incorporation of laser diodes and monomode fibre optics into an Electronic Speckle Pattern Interferometer (ESPI) has led to the development of a novel holographic surface contouring system. Height contours are obtained by switching between two laser wavelengths. Contour intervals from $0.5-5 \mathrm{~mm}$ have been generated by modulating the injection current of a single laser diode source and are demonstrated on an automotive disc brake hub.
\end{abstract}

\section{INTRODUCTION}

Electronic Speckle Pattern Interferometry (ESPI), also called TV holography, is widely used as a non-contact optical measurement technique for engineering structures [1]; examples include examining the deformation or vibration of body panels, engines and engine components in the automotive industry [2]. An essential feature of ESPI is that it provides simultaneous measurement over an extended area of the target surface. ESPI thus offers distinct advantages over point measurement techniques, such as laser velocimetry and interferometric vibrometry, and yields data unavailable from those techniques.

Although ESPI is a powerful tool its versatility is limited when implemented with conventional optical components; precise optical alignment is required and the system must essentially be confined to a vibration isolation table. Recently, the use of monomode optical fibre techniques has demonstrated that a substantial simplification of the optical arrangement, with a concomitant increase in flexibility [3], is possible. Applications involving test objects not mounted on an optical table are thus feasible.

ESPI techniques have also been applied to surface contouring; contouring, or profiling, is the measurement of variations in height and thus "surface profile" of the object under illumination. Several methods have been demonstrated based on projected fringe [4], reference surface [5] and two wavelength techniques $[1,6]$. 
Two wavelength technique offers the advantage of no moving parts in the interferometer assembly, and are therefore directly compatible with optical fibre techniques. Generally use has been made of neighbouring emission lines of an argon [1,] or krypton ion laser to generate high resolution $(5-30 \mu \mathrm{m})$ contour fringes. To obtain more versatility over the contour interval dye lasers have been used [1]. The use of ion lasers and ion pumped dye lasers is very costly and restricts the 'field' use of the techniques due to requirements of high electrical power, and in many cases water cooling.

In this paper we present a technique based on a diode laser coupled to monomode optical fibre allowing implementation of a complete solid state system. Wavelength modulation is achieved by modulating the injection current of the laser diode. The system is robust and comprises a compact transceiver, containing optics for object illumination, the reference beam and the CCD camera, linked to the laser diode by a flexible optical fibre cable.

\section{CONIOURING BY ESPI}

\subsection{ESPI}

ESPI may be regarded as in-line image plane holography where the photographic film has been replaced by a high sensitivity television camera. The basic experimental technique is shown in Figure 1. The configuration is analagous to that of a Mach-Zehnder interferometer where one mirror is replaced by the object under study. The input laser beam is amplitude divided and spatially filtered into a reference and object beam. The object beam illuminates the test object and is imaged using a camera lens onto the face of a CCD camera. The reference beam is also directed onto the face of the CCD camera such that the reference beam wavefront interferes with the wavefront from the object; the spatial resolution of the camera, typically $10-20 \mu \mathrm{m}$, is too small to resolve conventional holographic scale fringes.

Speckle is observed in the image plane of the camera where the speckle size, $\sigma$, is given by [1]

$$
\sigma=2.4 \lambda_{i} \mathrm{f} / \mathrm{D}
$$

where $\lambda_{i}$ is the laser wavelength, $f$ is the focal length of the imaging lens and $\mathrm{D}$ is the aperture size. Practically, the speckle size is adjusted to be approximately equal to the resolution of the TV camera.

The intensity in the camera image plane may be calculated by analogy with other two beam interferometers [8], and is found to be:

$$
I_{i}\left(r_{d}\right)=\frac{1}{2} I_{o i}\left[1+V_{i} \cos \left\{\frac{4 \pi h\left(r_{o}\right)}{\lambda_{i}}+\theta_{i}\right\}\right]
$$


where $I_{q}, V_{i}$ and $\theta_{i}$ are constants; $I_{0 i}$ is the mean intensity and $V_{i}$ is the visibility of the interference; $r$ and $r_{\text {a }}$ are position vectors defining points on the object and detector respectively.

The parameter $h$ thus represents the height (measured in the direction of the optical axis) of the object surface above some arbitrary reference plane (normal to the optical axis).

\subsection{Contouring}

Equation (2) shows that points of equal height on the object will exhibit equal intensities in the image, thus forming contours. Contours of equal intensity are separated by a height interval of $\lambda_{i} / 2$, which is too small for most practical purposes, especially given that the surface roughness is generally large on this scale. For this reason ESPI contour or profile fringes are normally obtained by illuminating the object at two wavelengths, $\lambda_{1}$ and $\lambda_{2}$, either simultaneously or sequentially. When the object is sequentially illuminated, the first image is recorded at $\lambda_{1}$ and then subtracted from the second image recorded at $\lambda_{2}$.

Using equation (2), and with suffices 1 and 2 representing illumination at wavelengths $\lambda_{1}$ and $\lambda_{2}$ leads to

$$
\Delta I=-I_{01} V_{1} A(h) B(h)
$$

where we have assumed that $I_{01} \simeq I_{02}, V_{1} \simeq V_{2}$, and that generally $\lambda_{1} \simeq \lambda_{2}$, and

$$
\begin{aligned}
& A(h)=\sin \frac{1}{2}\left(\frac{8 \pi h\left(r_{0}\right)}{\lambda_{1}}+\theta_{1}+\theta_{2}\right) \\
& B(h)=\sin \frac{1}{2}\left(\frac{4 \pi h\left(r_{0}\right)}{\lambda_{e f f}}+\theta_{1}-\theta_{2}\right)
\end{aligned}
$$

where

$$
\lambda_{\text {eff }}=\lambda_{1} \lambda_{2} /\left(\lambda_{2}-\lambda_{1}\right)=c / \Delta v
$$

where $\Delta v$ is the optical frequency difference between $\lambda_{1}$ and $\lambda_{2}$, and $c$ is the velocity of light.

In equation (3), $A(h)$ represents profile fringes of height interval $\lambda_{1} / 2$. These fringes are multiplied by $B(h)$, which is periodic with height interval $\lambda_{\text {eff }}$, where $\lambda_{\text {eff }} \gg \lambda_{1}$.

In a practical system, the signal of equation (3) is rectified so that $|\Delta I|$ is displayed. For most surfaces, roughness is large on the scale of $\lambda_{1} / 2$. 
Hence the display shows high speckle contrast in regions where $B(h)$ is large and dark regions where $B(h)$ is small. The dark regions are therefore profile contours of height interval $\lambda_{\text {eff }}$. By controlling $\lambda_{2}-\lambda_{1}$, any desired fringe interval can be produced.

In practise, the equalities

$$
I_{01}=I_{02} ; V_{1}=V_{2}
$$

are not satisfied exactly, and equation (8) becomes

$\Delta I=-I_{\circ 1} V_{1} A(h) B(h)+\frac{1}{2}\left(I_{\circ 1}-I_{\circ 2}\right)+\frac{1}{2}\left(I_{\circ 1} V_{1}-I_{02} V_{2}\right) \cos \left(\frac{4 \pi h\left(r_{0}\right)}{\lambda_{1}}+\theta_{1}\right)$

The term ( $\left.I_{01}-I_{\circ 2}\right)$ is a constant background which is readily removed by high pass filtering the $\Delta I$ signal. The last term in equation (8) represents profile fringes of interval $\lambda_{1} / 2$ which are unmodulated by $B(h)$, thus preventing regions corresponding to $B(h)=0$ from appearing perfectly dark and therefore reducing the visibility of the contour fringes.

\section{EXPERIMENIAL}

The experimental arrangement is shown in Figure 1. A $50 \mathrm{mw}$ laser diode (SONY SLD202V-3) operating in single longitudinal mode at $802 \mathrm{~nm}$ was used as the source. Temperature stabilisation to $\pm 0.02 \mathrm{~K}$ was achieved using a thermoelectric cooler. The beam is first collimated and then coupled into the fibre by a $\times 10$ microscope objective. The fibre was single mode at the laser wavelength and had a nominally circular core.

The fibre guided beam was amplitude divided using a directional coupler fabricated to give $95 \%$ of the light in the object arm and $5 \%$ in the reference arm. The output beam from the normally cleaved fibre end of the object arm was allowed to diverge onto the test surface placed approximately $0.5 \mathrm{~m}$ from the output fibre. The scattered light was collected by a camera lens and imaged onto the face of a CCD camera. The output from the reference beam recombines with the scattered light on the camera face.

The piezoelectric modulator facility, to control phase [9], was not used in these initial demonstration experiments.

\subsection{Electronic Signal Processing}

The video output from the camera was high pass filtered and rectified before displaying on a TV monitor. Contour fringes were produced in the following manner: the object under test was illuminated with wavelength $\lambda_{1}$ and the resulting speckle pattern stored. The wavelength was then shifted to $\lambda_{2}$ by modulation of the laser injection current. The resulting speckle pattern, generated at $\lambda_{2}$, was subtracted from the stored frame and the result displayed following basic signal processing. This procedure was 
repeated at TV frame rate with laser modulation synchronised to the camera trigger.

\section{RESULTS}

The system was arranged as described previously with the laser unmodulated. The camera lens was set at F-11 implying, from equation (1), a speckle size of $\sim 20 \mu \mathrm{m}$.

Figure 2 shows a speckle image of a stud in an automotive disc brake hub illuminated with a single wavelength. Figure 3 shows the contours generated by modulating the laser wavelength by $50 \mathrm{GHz}$, measured using a scanning Fabry-Perot interferometer, thus generating approximately $3 \mathrm{~mm}$ contour fringes. With increased modulation the laser can be made to oscillate between more widely spaced longitudinal modes resulting in narrower contour fringes, for example a frequency modulation of $350 \mathrm{GHz}$ gives rise to $0.5 \mathrm{~mm}$ contours.

For lower modulations, $<20 \mathrm{GHz}$, the laser wavelength changes continuously rather than between discrete modes. In this case the contours produced were too large $>10 \mathrm{~mm}$, to be easily observable with the field of view used, although broad fringes across the object were seen for modulations of approximately $20 \mathrm{GHz}$.

\section{SUMMARY}

A two wavelength holographic contouring technique based on laser diode emission frequency modulation and fibre optic components has been demonstrated. Illustrative experiments on a brake disc hub indicate that the technique offers distinct advantages compared to conventional ESPI contouring systems.

\section{REFERENCES}

[1] JONES, R. and WYKES, C., "Holographic and Speckle Interferometry," Cambridge University Press, (1983).

[2] DAVIES, J.C. and BUCKBERRY, C.H., "Application of electronic speckle pattern interferometry in automotive product development," VDI Berichte, (1986), 617,279-293.

[3] DAVIES, J.C. BUCKBERRY, C.H. JONES, J.D.C. and PANNELL, C.N., "Developments in electronic speckle pattern interferometry for automotive vibration analysis," SPIE, Porto, (1988) (in press).

[4] BERGQUIST, B.D. and MONTGOMERY, P., "Contouring by electronic speckle pattern interferometry, (ESPI)," SPIE, 599 (1985), 189-195.

[5] MACOVSKI, A. RAMSEY, S.D. and SCHAEFER, L.F., "Time-lapse interferometry and contouring using television systems," Appl.Opt.10, (1971), 2722-2727.

[6] FERCHER, A.F. HUGH, Z. and VRY, U., "Rough surface interferometry with a two-wavelength heterodyne speckle interferometer," Appl.Opt.24, (1985), 2181-2188.

[7] GASVIK, V.J., "Optical metrology," John wiley, Chp.6, (1987).

[8] STEEL, W.H., "Interferometry" Cambridge University Press, (1986).

[9] AI, C. and WYANT, J.C., "Effect of piezoelectric transducer non-linearity on phase shift interferometry" Appl.Opt.26, (1987), 1112-1116. 


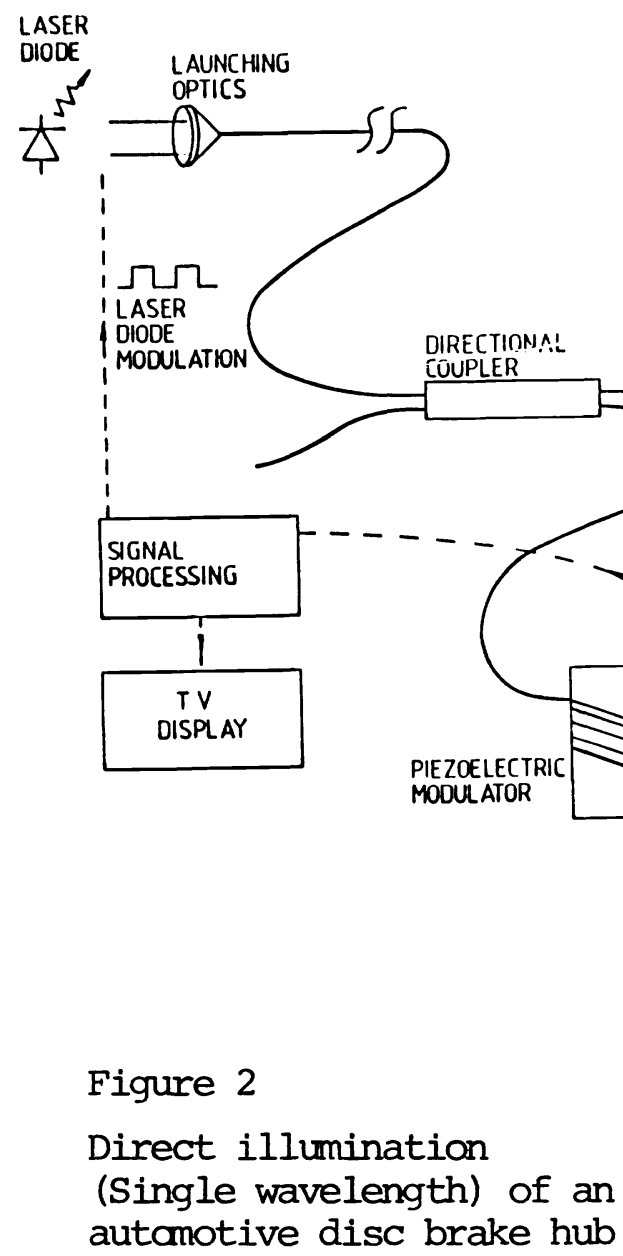

Figure 1 Experimental Arrangement autamotive disc brake hub

Figure 3

Contour fringes of disc brake hub : $\Delta v \simeq 50 \mathrm{GHz}$ 